\title{
Basic Creep-Fatigue Models Considering Cavitation
}

\author{
Rolf Sandström ${ }^{1} \mathbb{B}$
}

Received: 4 August 2021 / Accepted: 2 October 2021 / Published online: 30 October 2021

(c) The Author(s) 2021

\begin{abstract}
Cavitation plays a central role during creep-fatigue. During recent years, fundamental models for initiation and growth of creep cavities that do not involve any adjustable parameters have been developed. These models have successfully been used to predict creep rupture data for austenitic stainless steels again without using adjustable parameters. However, it appears that basic models have not yet been applied to creep-fatigue assessments. A summary of the fundamental cavitation models is given. A model for monotonous deformation is transferred to cyclic loading. The parameter values are kept except that the dynamic recovery constant is raised due to increased interactions between dislocations during cycling. This model is successfully compared with observed LCF and TMF hysteresis loops. A new model for cavity growth due to plastic deformation is presented. The model is formulated in such a way that the condition for constrained growth is automatically satisfied. In this way, it is avoided to overestimate the growth rate.
\end{abstract}

Keywords Creep $\cdot$ Plastic deformation $\cdot$ Hysteresis loop $\cdot$ Creep cavitation

\section{Introduction}

It has been known for a long time that creep cavities play an important role in connection with brittle creep rupture. When the amount of cavitation has reach a certain level, cracks are formed and rupture is initiated. The modelling of the formation of cavities has been a challenge. Several attempts have been made to formulate basic models based on classical nucleation theory, see for example (Raj and Ashby 1975). This approach has advantages and disadvantages. Even small variations in the parameter values give large differences in the results, which creates a lot of flexibility. The main disadvantage is that the model is not predictable. The model has to be calibrated to experimental data. However, there are other drawbacks as well. There is quite a strong stress dependence. Below a certain stress level, the cavitation nucleation rate is negligible and at higher stress levels, the nucleation rate is very fast. It is difficult to make this situation consistent with experimental data. In fact, it is well established for example for many steels that extensive cavity formation takes place at low stresses but to a more limited

Rolf Sandström

rsand@kth.se

1 Materials Science and Engineering, KTH Royal Institute of Technology, 10044 Stockholm, Sweden extent at high stresses. This is believed to be one of the main reasons why the creep ductility is typically lower at low operation stresses than at high ones.

Another common way to explain cavity nucleation is to assume that large stresses are present due to the existence of dislocation pile-ups (Yoo and Trinkaus 1982). Stresses in the GPa range are needed to form cavities. This would require long pile-ups that are rarely observed. However, high stresses can be generated with a shear crack. Riedel developed a shear crack model based on classical nucleation theory (Riedel 1984). The same problem as mentioned above with the stress dependence of cavity formation appears.

It is commonly assumed that cavity nucleation is due to grain boundary sliding (GBS). In particular, if particles are present in the grain boundaries, this is a natural assumption. The GBS displacement has been observed to be proportional to the creep strain. Finite-element simulations (FEM) of GBS give this proportionality as well (Ghahremani 1980). In addition, the number of nucleated cavities in steels and nickel base alloys has been observed to be proportional to the creep strain. These findings clearly show the narrow relation between GBS and cavity nucleation.

Thus with particles in the grain boundaries a simple explanation for the cavity formation is available. In addition, in some materials like pure copper with very few particles in the grain boundaries, extensive creep cavitation can occur. 
With modelling work, Lim demonstrated that cavity formation can take place in the presence of substructure (Lim 1987). In fact, cavities can form at intersections between cell or subboundaries and grain boundaries. He demonstrated that this process is thermodynamically feasible. It can be shown that the stress and strain dependence of the cavity formation that is predicted is consistent with observations. This is a most important result because it is no longer necessary to involve classical nucleation theory and the problems that are associated with it disappear. With the help of Lim's work, it is possible to formulate predictable cavitation models.

From the discussion above, it is evident that cavities can form in grain boundaries at particles or at intersections with subboundaries. These facts were expressed in the double ledge model (Sandström and Wu 2013). According to this model, the nucleation rate is proportional to the creep strain in agreement with observations. In addition, it gives the value of the proportionality constant. For the first time, a model was available that could quantitatively predict the cavity nucleation rate. This model will be further analysed below.

Basic models for cavity growth based on diffusion growth have been available for many years. Initially, these models significantly overestimated the growth rate. It was then realised that the cavity growth could not be faster that the creep rate of the surrounding material, which is referred to as constrained growth (Dyson 1976). Several models for constrained growth were formulated, see for example (Rice 1981). These models still tended to give too large growth rates. With the help of FEM analysis, a more precise expression for constrained growth could be obtained (He and Sandström 2016a). It gives results in good agreement with observations for austenitic stainless steels.

In addition to diffusion growth, strain-controlled growth can take place. A number of authors have presented model for cavity growth due to straining. They have often been used in empirical work on creep damage modelling. Many of these models can give quite large growth rates, often much larger that the observed ones. In these cases, the growth rates are larger than the creep deformation rate, i.e. the condition for constrained growth is not fulfilled. For diffusion-controlled growth, the authors have been quite careful to ensure that the criterion for constrained growth is satisfied, but this does not seem to be the case for strain-induced growth. However, there are models for strain-controlled growth that are consistent with constrained growth. One example will be given in the present paper.

Creep failure can be divided into ductile rupture and brittle rupture. The former mechanism is believed to be controlled by creep ductility exhaustion. Brittle rupture is initiated when the cavitated grain boundary area fraction exceeds a critical value. To predict creep rupture first, the creep deformation must be understood. The development of the dislocation structure, particle structure and the amount of elements in solid solution must be known to be able to compute dislocation strengthening, particle hardening and solid solution hardening. In addition, for brittle rupture, a quantitative description of cavity nucleation and growth must be available. Models for the microstructure development including cavity formation have successfully been generated. It has been demonstrated that they can quantitatively predict both creep ductile and brittle rupture times for austenitic stainless steels without the use of adjustable parameters ( $\mathrm{He}$ and Sandström 2017).

The principle described above is well established for monotonous deformation. However, for cyclic loading, basic models have been developed only to a limited extent. The purpose of the present paper is to formulate a basic model that can be used to compute different creep strain components during cyclic deformation. In addition, cavitation models are presented that can be applied to cyclic loading.

\section{Basic Model for Hysteresis Loops}

Numerous empirical models exist for describing LCF and TMF hysteresis loops. The measured data are fitted to mathematical expressions using a number of adjustable parameters. These expressions are in most cases just functions that are suitable to fit the data but are not based on a direct derivation starting from basic physical principles. The advantage with empirical models is that they are simple to use and it is easy to obtain an accurate description of the experimental data. However, there are essential drawbacks. Since many different expressions can be used to fit the data, it is not easy to safely find the one that represents the correct controlling mechanisms. When the operating mechanisms cannot be ascertained, any extrapolation to new conditions gives uncertain results, and extrapolation of experimental results is practically always desirable. Empirical models cannot be used to generalise results, except if a large amount of data is available for a given material. This is usually referred to as the empirical models are not predictable.

For creep and plastic deformation, basic models have been developed that are predictable, for a survey see (Sandström 2017). The models are derived from basic physical principles. It has been demonstrated that these models give results in quantitative agreement with observations for copper, aluminium, and stainless steels without involving adjustable parameters. In this text, such models are referred to as basic. Few attempts have been taken to derive basic models for cyclic deformation and for this reason, a model is presented here. It involves elastic, plastic and creep deformation. The plastic deformation flow curve can be represented with the Voce expression, which can be derived from basic dislocation 
mechanisms. The derivation of the expression and the parameters involved can be found in Sandström and Hallgren (2012):

$\sigma=\sigma_{y}+\left(\sigma_{\max \text { flow }}-\sigma_{y}\right)\left(1-e^{-\omega \varepsilon_{p l} / 2}\right)$

where $\sigma$ is the applied stress, $\varepsilon_{\mathrm{pl}}$ the plastic strain, $\sigma_{\mathrm{y}}$ the yield strength, $\sigma_{\max \text { flow }}$ the maximum flow stress, and $\omega$ the dynamic recovery constant. $\omega$ describes how fast the work hardening deviates from a linear behaviour. From Eq. (1), the plastic strain rate can be derived:

$\frac{d \varepsilon_{p l}}{d t}=\frac{2}{\omega\left(\sigma_{\max \text { flow }}-\sigma\right)} \frac{d \sigma}{d t}$.

The creep rate in the secondary stage $\dot{\varepsilon}_{\text {sec }}$ is given by Sandstrom (2012):

$\frac{d \varepsilon_{\mathrm{sec}}}{d t}=\frac{2 b c_{L}}{m} \frac{D_{\text {selfo } 0} b \tau_{L}}{k_{B} T}\left(\frac{\sigma}{\alpha G b}\right)^{3} e^{\frac{\sigma b^{3}}{k_{B} T}} e^{-\frac{Q_{\text {self }}}{R T}\left[1-\left(\frac{\sigma}{\sigma_{\text {max }}}\right)^{2}\right]} e_{\mathrm{P}}^{-\frac{Q_{\text {sol }}}{R T}}=h(\sigma)$,

where $T$ is the absolute temperature, $\sigma$ the applied stress, $D_{\mathrm{s} 0}$ the pre-exponential coefficient for self-diffusion, $Q_{\text {self }}$ the activation energy for self-diffusion, $k_{\mathrm{B}}$ Boltzmann's constant, $R_{G}$ the gas constant, $m$ the Taylor factor, $b$ Burger's vector, $\tau_{\mathrm{L}}$ the dislocation line tension, $\sigma_{i \max }$ the maximum flow stress, and $c_{\mathrm{L}}$ a work hardening constant. $\mathrm{Q}_{\text {sol }}$ is an additional contribution to the activation energy due to elements in solid solution. Due to the complexity of Eq. (3), a short hand notation $h(\sigma)$ is also introduced. At low stresses, Eq. (3) gives a stress exponent of about 3, but increases rapidly with increasing stress.

The elastic, plastic, and creep strain rate add up to the total external strain rate $\dot{\varepsilon}_{t o t}$. For computing the hysteresis loops, it is essential to take primary creep into account. The primary creep rate can be expressed as (Sui and Sandström 2018)

$\frac{d \varepsilon_{\text {prim }}}{d t}=h\left(2 \sigma_{s}-\sigma\right)$

where $\sigma_{\mathrm{s}}$ is the stress in the secondary stage that gives the external strain rate

$\frac{d \varepsilon_{t o t}}{d t}=h\left(\sigma_{s}\right)$

The sum of the elastic, plastic, and creep strain rate is equal to the external strain rate

$\frac{d \varepsilon_{e l}}{d t}+\frac{d \varepsilon_{p l}}{d t}+\frac{d \varepsilon_{p r i m}}{d t}=\frac{d \varepsilon_{t o t}}{d t}$,

where the elastic strain rate $\dot{\varepsilon}_{e l}$ is $\frac{d \varepsilon_{e l}}{d t}=\frac{d \sigma}{d t} \frac{1}{E}$

and $E$ is the elastic modulus. Equations (2), (4), (6) and (7) can now be combined and the stress rate that is used to compute the hysteresis loops is found:

$\frac{d \sigma}{d t}=\frac{1}{1 / E+2 /\left[\omega\left(\sigma_{\text {max flow }}-H\left(\dot{\varepsilon}_{\text {tot }}\right) \sigma\right)\right]}\left[\frac{d \varepsilon_{\text {tot }}}{d t}-h\left(2 \sigma_{s}-\sigma\right)\right]$.

The sign function $H$ has been introduced in Eq. (8) to make it valid for both the tension and compression going parts of the loop.

The main principle in the equations above is that the same parameter values as during monotonous loading should be used. The creep properties are often well characterised at high temperature. However, this is not always the case for tensile properties. The temperature dependence of the maximum flow stress follows approximately the temperature dependence of the elastic modulus in the following way (unpublished results):

$\sigma_{\max \text { flow }}(T)=\sigma_{\max \text { flow }}(R T)\left[\frac{E(T)}{E(R T)}\right]^{2}$,

where $T$ and $R T$ represent the value at temperature and room temperature, respectively. The $\omega$ value follows a similar behaviour. However, there is an additional effect. $\omega$ describes how often dislocations during deformation meet and annihilate if the burgers vectors match. During cycling, the dislocations meet much more often than during monotonous loading and consequently the annihilation rate is higher. Thus, the $\omega$ value must be higher. Each half cycle can in this respect be considered to be equivalent to the deformation until the uniform elongation is reached in the monotonous case. The total effect on $\omega$ can then be expressed as

$\omega(T)=\omega(R T) \frac{\varepsilon_{u}}{\varepsilon_{r}}\left[\frac{E(R T)}{E(T)}\right]^{2}$,

where $\varepsilon_{\mathrm{u}}$ is the uniform elongation during monotonous loading and $\varepsilon_{\mathrm{r}}$ the strain range during cycling. Notice that the effect of the temperature dependence of the elastic modulus is opposite for $\sigma_{\max \text { flow }}$ and $\omega$.

\section{Application of the Cycling Model}

In Eq. (8), an equation is given for the computation of a hysteresis loop. It is a basic model that is based on the same principles as applied during monotonous loading. Elastic, 
plastic and creep deformation are taken into account. The parameter values are the same as during monotonous loading. The only exception is the dynamic recovery constant $\omega$ that takes a higher value according to Eq. (10) for reasons explained in the previous section, namely that the dislocation during cyclic deformation encounters each other much more frequently than when the deformation rate is not reversed.

The use of Eq. (8) will now be illustrated for low cycle fatigue (LCF) of the $21 \mathrm{Cr} 11 \mathrm{Ni}$ austenitic stainless steel 253 MA. The steel has rare-earth metals (REM) additions to improve the oxidation resistance. Results for a hysteresis at $750{ }^{\circ} \mathrm{C}$ are compared to experimental data in Fig. 1 for the same conditions.

It is evident that the model gives a reasonable description of the experimental loop. Data for the investigated material can be found in Andersson et al. (2007).

To demonstrate the significance of the high $\omega$ value, a loop predicted without taking the loop factor $\varepsilon_{\mathrm{u}} / \varepsilon_{\mathrm{r}}$ into account is illustrated in Fig. 2. The other parameter values are unchanged.

It is not even possible to form a reasonably shaped loop with a low $\omega$.

Results will also be shown for another alloy PM2000. This alloy is a ferritic oxide dispersion strengthened (ODS) alloy with the approximate composition $20 \mathrm{Cr} 5 \mathrm{~A} 10.4 \mathrm{Ti} 0.5 \mathrm{Y}_{2} \mathrm{O}_{3}$ (Sandstrom and Andersson, 2003). A LCF loop is shown in Fig. 3. The appearance of the loop is quite different in comparison to that in Fig. 1: creep is much more significant due to the high temperature. This is apparent from the flat upper and lower parts of the loop. Since the creep deformation sets in at lower stresses, the "vertical" parts are controlled by the initial part of work hardening. They are straighter than in Fig. 1.

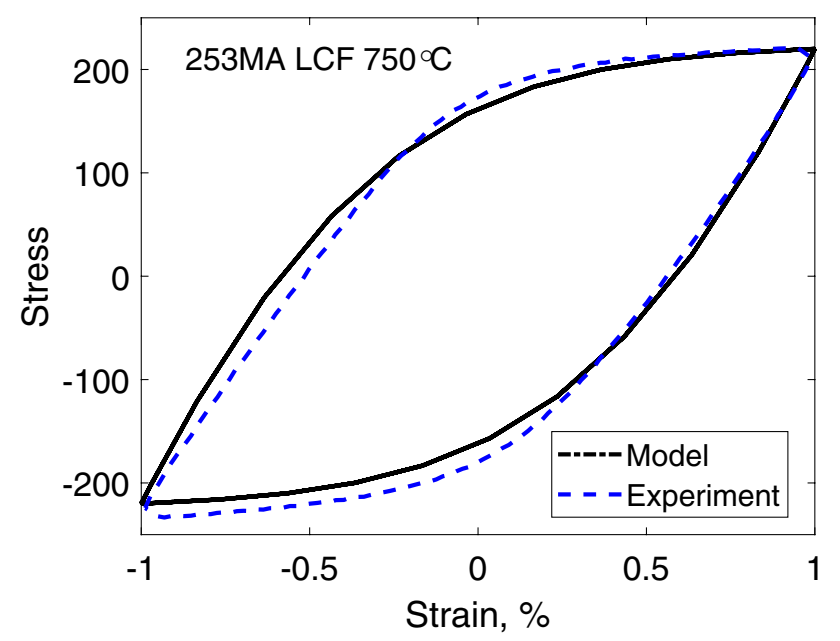

Fig. 1 Hysteresis loop for low cycle fatigue (LCF) of the austenitic stainless steel $253 \mathrm{MA}$ at $750{ }^{\circ} \mathrm{C}$. Experimental data are compared with the model in Eq. (8)

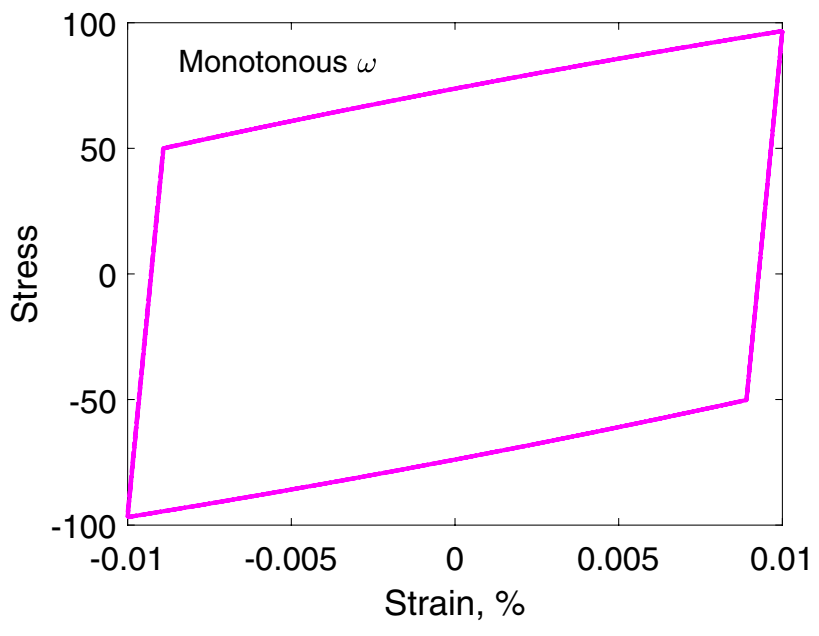

Fig. 2 Simulated hysteresis loop for low cycle fatigue (LCF) with the same parameter values except that a low $\omega(=15$ at room temperature) value characteristic of monotonous deformation is used

Another example of a LCF loop is presented in Fig. 4 for the same material as in Fig. 3

The lower strain rate has only a marginal influence on the loop.

A more severe test of the model is to compare it with a loop for thermo-mechanical fatigue (TMF). An example is shown in Fig. 5 for PM2000. The thermal cycling is between 800 and $1200{ }^{\circ} \mathrm{C}$ with strain and temperature in phase. Also the TMF loop can be described well.

The loops in Figs. 3, 4, and 5 have been modelled previously with empirical equations (Sandstrom and Andersson 2003) where adjustable parameters were used. The results in the present paper demonstrate that hysteresis loops can be described with basic models. In this way,

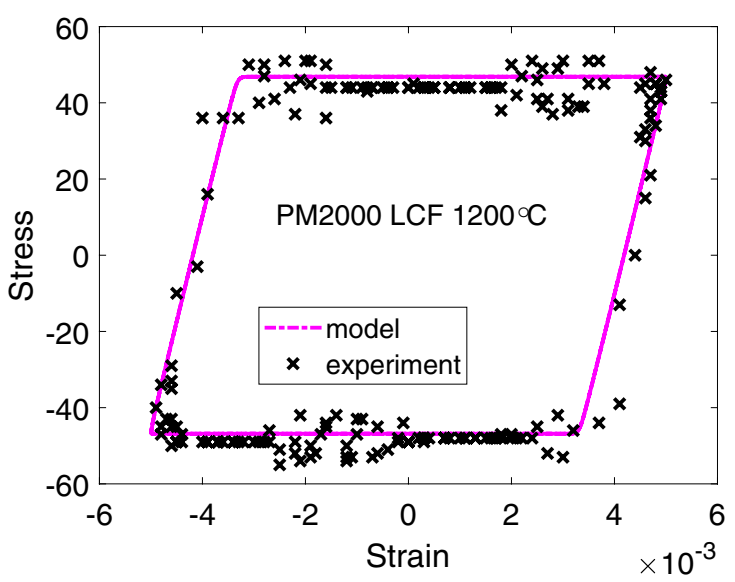

Fig. 3 Hysteresis loop for low cycle fatigue (LCF) of the ferritic ODS alloy at $1200{ }^{\circ} \mathrm{C}$. Strain rate $7 \times 10^{-4} 1 / \mathrm{s}$. Experimental data are compared with the model in Eq. (8) 


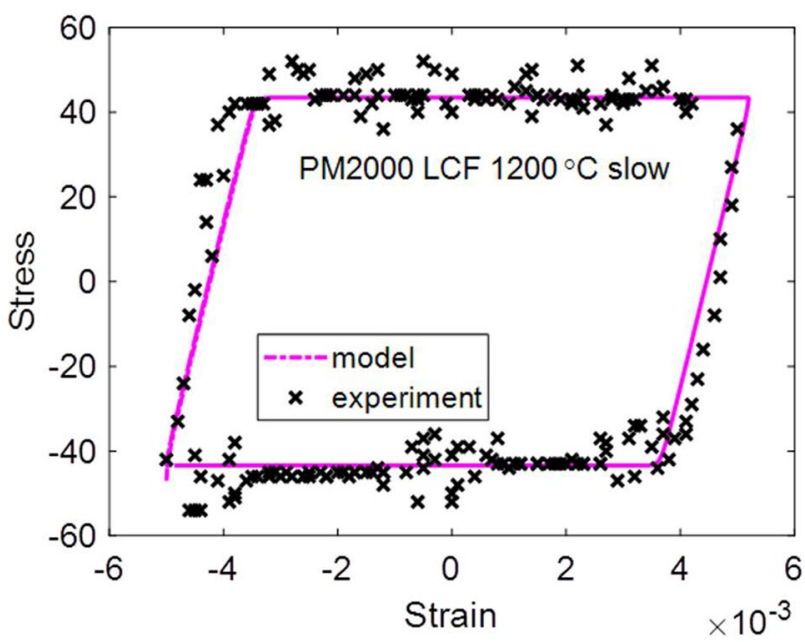

Fig. 4 Hysteresis loop for low cycle fatigue (LCF) of the ferritic ODS alloy PM2000 at $1200{ }^{\circ} \mathrm{C}$. A lower strain rate of $5 \times 10^{-5} 1 / \mathrm{s}$ than in Fig. 3 is used. Experimental data are compared with the model in Eq. (8)

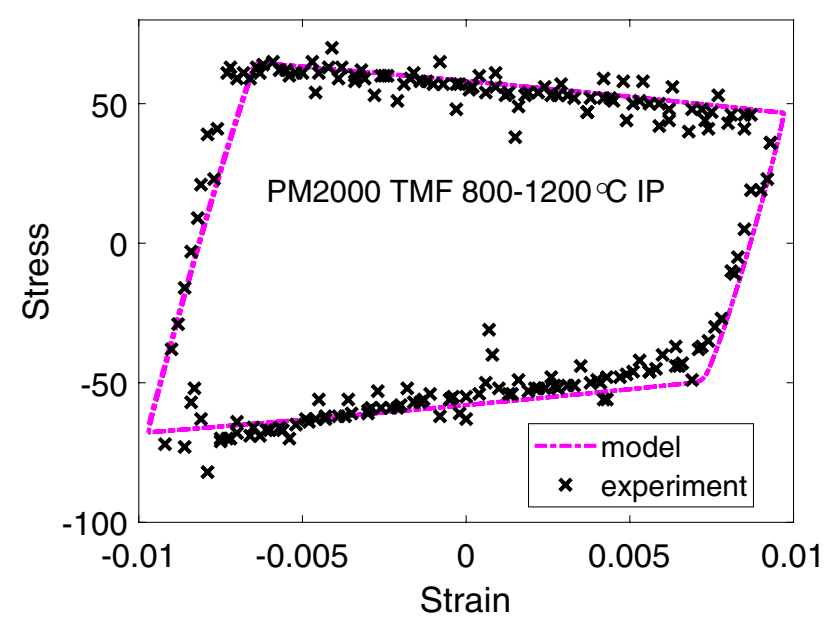

Fig. 5 Hysteresis loop for thermo-mechanical fatigue (TMF) of the ferritic ODS alloy PM2000 between 800 and $1200{ }^{\circ} \mathrm{C}$ in phase. Strain rate $5 \times 10^{-5} 1 / \mathrm{s}$. Experimental data are compared with the model in Eq. (8)

it is possible to predict the creep damage in more general cases and not just associated with measured loops. In the past, it has been assumed that the shape of hysteresis loops is due to the presence of residual stresses as in the Masing model or a complex distribution of yield strengths (Skelton et al. 1997). The present results show that this is not necessarily the case and cyclic and monotonous deformation can be handled in a similar way.
Nucleation and Growth of Creep Cavities

\section{Cavity Nucleation}

It is well established that nucleation of cavities takes place by grain boundary sliding (GBS). Sliding grain boundaries open cavities at particles and subboundary-grain boundary junctions as explained in the introduction. In spite of this, only recently basic quantitative models for nucleation of cavities have been presented directly related to GBS. Only a brief survey of these models will be given here since a detailed review can be found elsewhere (Sandström and He 2017).

The starting point is that the GBS displacement $u_{\mathrm{GBS}}$ is proportional to the creep strain

$u_{\mathrm{GBS}}=C_{\mathrm{s}} \varepsilon$.

FEM modelling has given the value of the constant $C_{\mathrm{s}}$ (Ghahremani 1980):

$C_{\mathrm{s}}=\dot{u}_{\mathrm{GBS}} / \dot{\varepsilon}=\frac{3 \phi}{2 \xi} d_{\mathrm{g}}$,

where $d_{\mathrm{g}}$ is the grain size, $\phi=0.15-0.33$ (the value increases with the creep stress exponent) and $\xi \approx 1.4$ are constants. To relate the cavity nucleation to the creep strain, Sandström and $\mathrm{Wu}$ introduced the so-called double ledge model (Sandström and Wu 2013). According the ideas in Lim's model, nucleation is assumed to take place when a subboundary on one side of a sliding grain boundary meets a particle or another subboundary on the opposite side. This gives the following rate of cavitation nucleation:

$\frac{d n_{\mathrm{cav}}}{d t}=\frac{0.9 C_{s}}{d_{\text {sub }}}\left(\frac{g_{\text {sub }}}{d_{\text {sub }}^{2}}+\frac{g_{\text {part }}}{\lambda^{2}}\right) \dot{\varepsilon}=B \dot{\varepsilon}$,

where $n_{\text {cav }}$ is the number of cavities nucleated per unit grain boundary area, and $d_{\text {sub }}$ is the subgrain diameter. $d_{\text {sub }}$ is inversely proportional to the dislocation stress that is in general close to the applied stress. $\lambda$ is the interparticle spacing in the grain boundary. $\mathrm{g}_{\text {part }}$ is the fraction of particles in the grain boundaries where cavities are nucleated. $g_{\text {sub }}$ is the corresponding fraction of subboundary-grain boundary junctions where cavities are formed. The factor 0.9 takes into account the averaging over different orientations.

The expression in Eq. (13) has been compared successfully with observations for a range of austenitic stainless steels (He and Sandström 2016b). In these cases, $g_{\text {sub }}$ and $\mathrm{g}_{\text {part }}$ could be assumed to be equal to unity but that cannot always be considered to be the case. 


\section{Diffusion-Controlled Cavity Growth}

Expressions for diffusion-controlled growth of cavities have been derived by a number of authors. Early expressions grossly overestimated the growth rate. It was then realised that the growth rate cannot be larger than the creep deformation rate of the surrounding materials, which is referred to as constrained growth (Dyson 1976). An expression for constrained growth was derived by Rice (Rice 1981):

$\frac{d R_{\mathrm{cav}}}{d t}=2 D_{0} K_{f}\left(\sigma_{\mathrm{red}}-\sigma_{0}\right) \frac{1}{R_{\mathrm{cav}}^{2}}$

where

$\sigma_{\text {red }}=\sigma_{0}+\frac{1}{\frac{1}{\sigma_{\text {appl }}}+\frac{32 D_{0} K_{f}}{\lambda_{\text {cav }}^{2} d_{\mathrm{g}} \beta \dot{\varepsilon}\left(\sigma_{\text {app }}\right)}}$.

$R_{\text {cav }}$ is the cavity radius in the grain boundary plane, $d R_{\text {cav }} / d t$ its growth rate, $\sigma_{0}$ the sintering stress, $2 \gamma_{\mathrm{s}}$ $\sin (\alpha) / R_{\text {cav }}$, where $\gamma_{\mathrm{s}}$ is the surface energy of the cavity per unit area and $\alpha$ the cavity tip angle. The presence of the sintering stress $\sigma_{0}$ ensures that only cavities that are larger than a critical size grow. $\delta$ the grain boundary width, $D_{\mathrm{GB}}$ the grain boundary self-diffusion coefficient, and $\Omega$ the atomic volume are combined into a grain boundary diffusion parameter $D_{0}, D_{0}=\delta D_{\mathrm{GB}} \Omega / k_{\mathrm{B}} T . k_{\mathrm{B}}$ is the Boltzmann's constant and $T$ the absolute temperature. $\beta$ is a material constant $(\beta=1.8$ for homogeneous materials), and $K_{\mathrm{f}} \approx 0.2$ is a constant. In unconstrained growth, the applied stress $\sigma_{\text {appl }}$ appears in Eq. (14) instead of the reduced stress $\sigma_{\text {red. }}$.

Although Eq. (14) represents a much improved model in comparison to unconstrained growth, the equation still tends to overestimate the growth rate. He and Sandström reanalysed the growth rate with the help of FEM modelling to find the extent of the region around a cavity where the deformation rate was affected (He and Sandström 2016a). A pillar of height $h$ and width corresponding to the grain size $d_{\mathrm{g}}$ was set up. In this pillar, the creep deformation in the loading direction $z$ can be expressed as

$\frac{d z}{d t}=4 \pi D_{0} K_{f}\left(\sigma_{\text {red }}-\sigma_{0}\right) n_{c a v}+h \dot{\varepsilon}\left(\sigma_{\text {red }}\right)=h \dot{\varepsilon}\left(\sigma_{\text {appl }}\right)$

where $\dot{\varepsilon}\left(\sigma_{\text {red }}\right)$ and $\dot{\varepsilon}\left(\sigma_{\text {appl }}\right)$ are the creep rates at the reduced and applied stress, respectively. In the first expression for $\dot{z}$, the first term is the volume growth rate of a cavity multiplied by the number of cavities per unit grain boundary area. The second term is the creep displacement of the pillar at the reduced stress. The second expression for $z$ is the displacement of the surrounding material at the applied stress. The result of the FEM analysis was that $h \approx 2 R_{\text {cav. }}$ Using this result, Eq. (16) can be rewritten as
$2 \pi D_{0} K_{f}\left(\sigma_{\text {red }}-\sigma_{0}\right) n_{c a v} / R_{c a v}+\dot{\varepsilon}\left(\sigma_{\text {red }}\right)=\dot{\varepsilon}\left(\sigma_{\text {appl }}\right)$.

Equation (17) has to be solved by iteration. Using Eq. (17) for the reduced stress in Eq. (14), a lower and improved growth rate is obtained in good agreement with observations for austenitic stainless steels (He and Sandström 2016a).

\section{Strain-Controlled Cavity Growth}

In addition, due to diffusion, creep cavities can grow due to straining. There exist several basic models in the literature for cavity growth due to creep straining. Unfortunately, few of them obey the constrained growth criterion. As a consequence, the models can predict growth rates that are much larger than the observed ones. This is for example the case when diffusion control first forms micron sized cavities and then strain control is applied. For this reason, a new simple model for strain-controlled growth will be presented that automatically satisfy the constrained growth criterion.

In the grain boundary plane, creep cavities are often elongated. When cavities have nucleated for example around particles, the cavities continue to be exposed to GBS. As a consequence, they see shearing strains that make the cavities grow. A simple assumption is that the growth rate is equal to the GBS rate. This will result in a cavity radius of, see Eq. (11):

$R_{\text {cav }}=C_{\mathrm{s}} \varepsilon$,

where $C_{\mathrm{s}}$ is again given by Eq. (12). Equation (18) is compared to experiments for a $12 \mathrm{CrMo}$ steel and an austenitic stainless steel TP347 (17Cr12NiNb) in Fig. 6.

Equation (18) gives an approximate description of the data for the two materials. There are very limited data in the literature to make further comparisons. Since the constant is proportional to the grain size, large growth rates are predicted with large grain sizes, and those predictions may not always be realistic.

$R_{\text {cav }}$ according Eq. (18) is directly controlled by the creep strain and the condition for constrained growth is automatically satisfied. However, it is important to recognise that there is no margin in this respect. The criterion for constrained growth is just fulfilled. The same applies to Eq. (14) for diffusion-controlled growth. This means that Eq. (18) cannot be combined with any expression diffusioncontrolled growth, and Eq. (14) cannot be used together with plastic straining without violating the criterion for constrained growth.

\section{Cavitation During Cycling}

Creep cavitation is known to take place during LCF and TMF. One can expect that the number of cavities formed is 

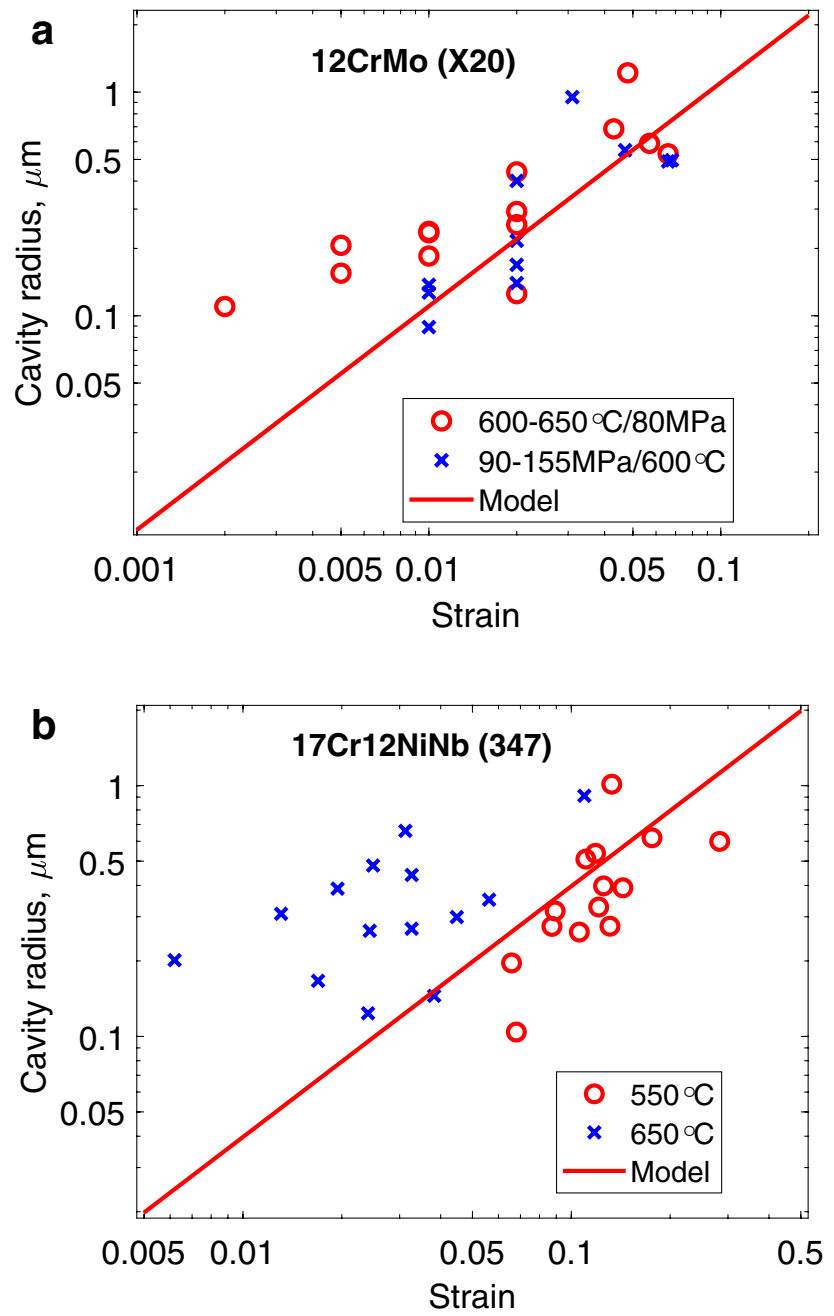

Fig. 6 Cavity radius versus strain. Model according Eq. (18); (a) 12CrMo steel (X20). Data from (Wu and Sandstrom 1995); (b) TP347 (17Cr12NiNb). Data from (Needham and Gladman 1980)

proportional to the creep strain during cycling. It is natural to assume that there is an effective creep strain consisting of two parts that contribute. There is a creep strain range $\Delta \varepsilon_{\mathrm{rc}}$ that is a part of the plastic strain range. This gives a creep strain of $2 \Delta \varepsilon_{\mathrm{rc}}$ in each cycle. During TMF, there is not a complete match between the creep strain in the tension and compression going part of the cycle. This unbalance generates a strain component $\Delta \varepsilon_{\text {unc }}$. If hold times are present in the cycle, there are additional contributions to $\Delta \varepsilon_{\text {unc }}$. With these strain components, the cavity nucleation rate is assumed to have a similar form to Eq. (13) and can be expressed as

$n_{\text {cav }}=B\left(2 f_{n} \Delta e_{r c}+\left|\Delta \varepsilon_{\text {unc }}\right|\right)$.

If both the tension and compression going part were equally effective in generating cavities, the constant $f_{\mathrm{n}}$ would be equal to unity. However, it is likely that a significant fraction of the cavities are closed when the loading direction is reversed, which means that $f_{\mathrm{n}}$ is smaller than unity.

Diffusion-controlled growth is not likely to be of importance for the short testing times that are used in typical laboratory testing. However, strain control can be significant. Essentially the same arguments as during nucleation can be used, i.e. the two strain components $\Delta \varepsilon_{\mathrm{rc}}$ and $\Delta \varepsilon_{\mathrm{unc}}$ are of importance. If the cavity growth is assumed to follow Eq. (18), a plausible form of the growth takes the form

$R_{\text {cav }}=C_{s}\left(2 f_{R} \Delta e_{r c}+\left|\Delta \varepsilon_{\text {unc }}\right|\right)$.

The constants $f_{\mathrm{n}}$ and $f_{\mathrm{R}}$ are not necessarily identical. Until models for $f_{\mathrm{n}}$ and $f_{\mathrm{R}}$ are developed, Eqs. (19) and (20) should be considered as empirical relations.

With the help of the models in "Basic Model for Hysteresis Loops", the creep strain components $\Delta \varepsilon_{\mathrm{rc}}$ and $\Delta \varepsilon_{\mathrm{unc}}$ can be computed directly. Unfortunately, no data have been found in the literature that can be used to verify the expressions (19) and (20). They should, therefore, be considered as tentative. Some indirect support of the models are available since it has been found that the creep strain during hold times can be correlated to the number of cycles to failure, see for example (Sandström et al. 1989) and (Nilsson and Sandström 1988).

\section{Discussion}

In recent years, basic models for creep deformation have been developed (Sandström 2017). The meaning of "basic" in this context is that the models are derived from fundamental physical principles and do not involve any adjustable parameters. This is important in many technical applications because it makes the models predictable and new results can be derived even if they are not close to the experimental data. Empirical models with a range of adjustable parameters still dominate creep research and creep applications. In addition to their lack of predictability, the results are uncertain if they are used to identify operating mechanisms.

Basic models for nucleation and growth of creep cavities have been formulated and further developed in the last few years (Sandström and He 2017). They have been verified experimentally for creep deformation in tension. Attempts to develop corresponding models for cyclic loading have been quite limited. The need for basic models is in fact larger for cyclic than for monotonous deformation. The reason is simply that the testing times in cyclic loading experiments are typically much shorter than for example creep in tension. Thus, much more extensive extrapolation is required for cyclic loading, and to make that in a reasonably safe way, 
basic models are needed. It is possible to extrapolate with the help of empirical models but a large amount of data for a number of conditions must be at hand for a given material.

To establish basic models for cavitation for cyclic loading, two steps are essential. The first step is to develop models for the cyclic deformation. The second step is to formulate equations for cavity nucleation and growth. The natural starting point is to use as much of the basic models for monotonous loading and analyse if they can be applied with appropriate changes. This is also precisely what is attempted in the present paper.

Empirical descriptions of cyclic deformation follow essentially two tracks. The first track involves fitting a mathematical expression such as the Ramberg-Osgood expression to the observed hysterical loop. The second track is to fit a multi-parameter formula such as the Masing model to the data (Skelton et al. 1997). These approaches easily give a good fit to the data, but they are not derived from physical principles, and they are associated with all the limitations mentioned above for empirical models.

Basic models are available for plastic and creep deformation with parameter values that are well defined (Sandström 2017). As demonstrated in "Application of the Cycling Model", if the same parameters are used as for monotonous loading, these models are not directly applicable to cyclic loading. The main reason for this was identified in "Basic Model for Hysteresis Loops", namely the value of the dynamic recovery parameter $\omega$. This parameter describes how fast dislocations interact and find dislocations with opposite burgers vectors and annihilate. The value of $\omega$ determines how much the work hardening deviates from a linear behaviour. With $\omega=0$, a stress strain curve would be straight. $\omega$ plays an important role also for the creep deformation. For example, $\omega$ strongly influences tertiary creep and the effect of cold working (Sandström 2017), but that is not the topic of the present paper. During cyclic deformation, the dislocations encounter each other much more frequently, which results in a much higher value of $\omega$. This was quantitatively modelled in "Basic Model for Hysteresis Loops". With the raised value of $\omega$, quite reasonable representations of hysteresis loops were obtained as shown in "Application of the Cycling Model".

Extensive cavitation is known to take place during cyclic loading at elevated temperatures. Unfortunately, quantitative information is available only to a limited extent. It is natural to assume that the same principles control nucleation and growth of cavities as during monotonous deformation. Thus, the nucleation rate is proportional to the creep strain rate. Since the total plastic deformation is larger during cyclic loading, it is expected that cavity growth mainly take place due to plastic deformation and not due to diffusion-controlled growth. A new simple model for straincontrolled growth is presented in "Nucleation and Growth of
Creep Cavities". It is directly related to the amount of grain boundary sliding. It is believed that the cavity nucleation and growth are controlled both by the creep strain range during cycling as well as any additional creep strain during hold times or due to the unbalance in the TMF cycles. These creep strains can be directly computed with the models in "Basic Model for Hysteresis Loops". Tentative expressions were formulated for cavity nucleation and growth but they need experimental verification.

\section{Conclusions}

There is great need for basic models for cyclic deformation and for cavitation under cyclic conditions. With basic models, extrapolation to new conditions can be performed in a much safer way. The improved possibilities of extrapolation to longer times are particularly important for cyclic deformation due to the often limited testing times. In the present paper, a first attempt to formulate basic models for cyclic deformation is taken.

1. Well-developed models for monotonous deformation are available. Such models have been reformulated to adapt to cyclic loading.

2. Most parameter values in the models for monotonous deformation are taken over. However, the value of the dynamic recovery parameter $\omega$ has to be changed and a model is presented for that. The reason is that the dislocations encounter each other much more frequently during cyclic loading and that gives a much higher $\omega$ value.

3. With the raised $\omega$ value, the proposed model for cyclic deformation has been compared successfully to LCF and TMF hysteresis loops for an austenitic stainless steel 253 MA and a ferritic oxide dispersion strengthened alloy PM2000.

4. Basic models for cavitation for monotonous loading are briefly reviewed. The nucleation rate is proportional to the creep strain. During cyclic loading, plastic strain rather than diffusion-controlled growth is expected to be of importance. A new simple model for growth due to plastic straining has been presented. Contrary to several such models in the literature, it satisfies the condition for constrained growth. This is essential to avoid overestimating the growth rate.

Funding Open access funding provided by Royal Institute of Technology. 
Open Access This article is licensed under a Creative Commons Attribution 4.0 International License, which permits use, sharing, adaptation, distribution and reproduction in any medium or format, as long as you give appropriate credit to the original author(s) and the source, provide a link to the Creative Commons licence, and indicate if changes were made. The images or other third party material in this article are included in the article's Creative Commons licence, unless indicated otherwise in a credit line to the material. If material is not included in the article's Creative Commons licence and your intended use is not permitted by statutory regulation or exceeds the permitted use, you will need to obtain permission directly from the copyright holder. To view a copy of this licence, visit http://creativecommons.org/licenses/by/4.0/.

\section{References}

Andersson HCM, Sandstrom R, Debord D (2007) Low cycle fatigue of four stainless steels in 20\% CO-80\% H-2. Int J Fatigue 29:119-127

Dyson BF (1976) Constraints on diffusional cavity growth rates. Met Sci 10:349-353

Ghahremani F (1980) Effect of grain boundary sliding on steady creep of polycrystals. Int J Solids Struct 16:847-862

He J, Sandström R (2016a) Creep cavity growth models for austenitic stainless steels. Mater Sci Eng A 674:328-334

He J, Sandström R (2016b) Formation of creep cavities in austenitic stainless steels. J Mater Sci 51:6674-6685

He J, Sandström R (2017) Basic modelling of creep rupture in austenitic stainless steels. Theoret Appl Fract Mech 89:139-146

Lim LC (1987) Cavity nucleation at high temperatures involving pileups of grain boundary dislocations. Acta Metall 35:1663-1673

Needham NG, Gladman T (1980) Nucleation and growth of creep cavities in a type 347 steel. Metal Science 14:64-72

Nilsson JO, Sandström R (1988) Influence of temperature and microstructure on creep-fatigue of alloy $800 \mathrm{H}$. High Temp Technol 6:181-186

Raj R, Ashby MF (1975) Intergranular fracture at elevated temperature. Acta Metall 23:653-666

Rice JR (1981) Constraints on the diffusive cavitation of isolated grain boundary facets in creeping polycrystals. Acta Metall 29:675-681
Riedel H (1984) Cavity nucleation at particles on sliding grain boundaries. A shear crack model for grain boundary sliding in creeping polycrystals. Acta Metal 32:313-321

Sandstrom R (2012) Basic model for primary and secondary creep in copper. Acta Mater 60:314-322

Sandstrom R, Andersson HCM (2003) Modelling of hysteresis loops during thermomechanical fatigue. Modelling of hysteresis loops during thermomechanical fatigue. ASTM Special Technical Publication, pp 31-44

Sandström R, Engstrom J, Nilsson JO, Nordgren A (1989) Elevated temperature low-cycle fatigue of the austenitic stainless steels type 316 and 253MA. Influence of microstructure and damage mechanisms. High Temp Technol 7:2-10

Sandström R (2017) Fundamental models for the creep of metals. In: Tanski T (ed) creep. Intech

Sandström R, Hallgren J (2012) The role of creep in stress strain curves for copper. J Nucl Mater 422:51-57

Sandström R, He J (2017) Survey of Creep cavitation in fcc METALS. In: Tanski WBT (ed) study of grain boundary character. Intech, pp 19-42

Sandström R, Wu R (2013) Influence of phosphorus on the creep ductility of copper. J Nucl Mater 441:364-371

Skelton RP, Maier HJ, Christ HJ (1997) The Bauschinger effect, Masing model and the Ramberg-Osgood relation for cyclic deformation in metals. Mater Sci Eng, A 238:377-390

Sui F, Sandström R (2018) Basic modelling of tertiary creep of copper. J Mater Sci 53:6850-6863

Wu R, Sandstrom R (1995) Creep cavity nucleation and growth in 12cr-Mo-V Steel. Mater Sci Tech Ser 11:579-588

Yoo MH, Trinkaus H (1982) Crack and cavity nucleation at interfaces during creep, metallurgical transactions. A Phys Metal Mater Sci 14A:547-561

Publisher's Note Springer Nature remains neutral with regard to jurisdictional claims in published maps and institutional affiliations. 